\title{
ON SIMPLE REDUCIBLE LIE ALGEBRAS OF DEPTH TWO
}

\author{
THOMAS B. GREGORY
}

\begin{abstract}
We show that under certain circumstances simple finite-dimensional reducible graded Lie algebras of the form $L_{-2} \oplus L_{-1} \oplus L_{0} \oplus L_{1} \oplus \cdots \oplus L_{k}$ can be given irreducible transitive gradations of the form $M_{-1} \oplus M_{0} \oplus \cdots \oplus M_{[k / 2]}$.
\end{abstract}

In [1], the present author classified the simple finite-dimensional irreducible graded Lie algebras over an algebraically closed field of characteristic $p>5$ which have the form

$$
L_{-2} \oplus L_{-1} \oplus L_{0} \oplus L_{1} \oplus \cdots \oplus L_{k}
$$

where $L_{0}$ is classical and reductive and $k \geqslant 3$. In this paper, we consider certain reducible Lie algebras $L$ of the form (1). In them, we show how to construct an irreducible, transitive gradation

$$
L=M_{-1} \oplus M_{0} \oplus \cdots \oplus M_{[k / 2]} \text {. }
$$

THEOREM. Let $L$ be a simple finite-dimensional reducible graded Lie algebra of the form (1) over a field of any characteristic, and suppose that $L_{0}$ contains no nonzero abelian ideal which annihilates $L_{-2}$ and that $L_{-1}=S+T$, where $S$ and $T$ are proper $L_{0}$-submodules of $L_{-1}$. Then $S$ and $T$ are irreducible abelian $L_{0}$-submodules, and $L_{-1}=S \oplus T$. In addition, $L$ possesses an irreducible transitive gradation of the form (2).

We will prove this theorem by means of a series of lemmas. We begin by restating the first three lemmas of [1]. Their proofs there are valid in this setting, also.

LEMMA 1. If $L$ is a simple graded Lie algebra of the form (1), then $L_{-2}$ is an irreducible $L_{0}$-module. Furthermore, $\left[L_{-1}, L_{-1}\right] \neq\{0\}$, so $\left[L_{-1}, L_{-1}\right]=L_{-2}$; also, $L_{-1}=\left[L_{-2}, L_{1}\right]$. Lastly, $\left[L_{-1}, x\right] \neq\{0\}$ for all $x \notin L_{-2}$.

LEMMA 2. If $L$ is a simple graded Lie algebra of the form (1), then $L_{k}$ is an irreducible $L_{0}$-module, and $L_{j-1}=\left[L_{j}, L_{-1}\right]$, for $-1<j<k$.

Lemma 3. We have $\left[L_{-2}, L_{i}\right] \neq\{0\}, 0 \leqslant i<k$, and $\left[L_{0}, L_{k}\right] \neq\{0\}$.

LEMMA 4. $L_{-1}$ contains no proper nonabelian $L_{0}$-submodule.

Proof. Let $N$ be a nonzero nonabelian $L_{0}$-submodule of $L_{-1}$. Then $[N, N]$ is a nonzero $L_{0}$-submodule of the irreducible $L_{0}$-submodule $L_{-2}$, so $[N, N]=L_{-2}$.

Received by the editors August 18, 1980 and, in revised form, November 17, 1980.

AMS (MOS) subject classifications (1970). Primary 17B20.

() 1981 American Mathematical Society 0002-9939/81/0000-0407/\$02.25 
Then we have by Lemma 1 that $L_{-1}=\left[L_{-2}, L_{1}\right]=\left[[N, N], L_{1}\right] \subseteq\left[\left[N, L_{1}\right], N\right]+$ $\left[N,\left[N, L_{1}\right]\right] \subseteq\left[L_{0}, N\right]+\left[N, L_{0}\right] \subseteq N$, so that $N=L_{-1}$. Q.E.D.

Lemmas 5 through 9 are valid for any simple finite-dimensional $L$ of the form (1) where $L_{0}$ contains no nonzero abelian ideal which annihilates $L_{-2}$ and where $L_{-1}$ contains a proper $L_{0}$-submodule $S$.

Lemma 5. Let $X$ be an $L_{0}$-submodule of $L_{0}$ such that $\left[L_{-1}, X\right]$ is abelian and $\left[L_{-2}, X\right]=\{0\}$. Then $X=\{0\}$.

Proof. We have $\left[L_{-1},\left[\left[L_{-1}, X\right], X\right]\right] \subseteq\left[\left[L_{-1},\left[L_{-1}, X\right]\right], X\right]+\left[\left[L_{-1}, X\right]\right.$, $\left.\left[L_{-1}, X\right]\right] \subseteq\left[L_{-2}, X\right]+\{0\}=\{0\}$. Thus, by Lemma $1,\left[\left[L_{-1}, X\right], X\right]=\{0\}$. Then $\left[L_{-1},[X, X]\right] \subseteq\left[\left[L_{-1}, X\right], X\right]+\left[X,\left[L_{-1}, X\right]\right]=\{0\}$, so $[X, X]=\{0\}$. Since $L_{0}$ is assumed to contain no nonzero abelian ideal which annihilates $L_{-2}$, we must have $X=\{0\}$. Q.E.D.

LEMMA 6. $\left[S,\left[S, L_{2}\right]\right]=\{0\}$.

Proof. We have that $\left[L_{-1},\left[S,\left[S, L_{2}\right]\right] \subseteq\left[\left[L_{-1}, S\right],\left[S, L_{2}\right]\right]+\right.$ $\left[S,\left[\left[L_{-1}, S\right], L_{2}\right]\right]+\left[S,\left[S,\left[L_{-1}, L_{2}\right]\right]\right] \subseteq\left[S,\left[\left[L_{-1}, S\right], L_{2}\right]\right]+\left[S, L_{0}\right]+\left[S, L_{0}\right]$ $\subseteq S$, which is abelian by Lemma 4. Furthermore, $\left[L_{-2},\left[S,\left[S, L_{2}\right]\right]\right]=$ $\left[S,\left[S,\left[L_{-2}, L_{2}\right]\right]\right] \subseteq\left[S,\left[S, L_{0}\right]\right] \subseteq[S, S]=\{0\}$. Thus, by Lemma $5,\left[S,\left[S, L_{2}\right]\right]=$ \{0\}. Q.E.D.

LEMMA 7. $S$ is contained in a maximal $L_{0}$-submodule $U$ of $L_{-1}$ such that $U=\operatorname{Ann}_{L_{-1}} U$.

Proof (Referee). Let $U$ be a maximal proper $L_{0}$-submodule of $L_{-1}$ containing $S$. Then by Lemma $4,\{0\}=[U, U]=[U, S]$, so $U \subseteq A_{L^{-1}} U \subseteq A_{L^{\prime-1}} S$. Since $\left[L_{-1}, S\right] \neq\{0\}$ (by Lemma 1 ), the maximality of $U$ forces $U=\operatorname{Ann}_{L_{-1}} U=$ $\mathrm{Ann}_{L_{-1}} S$. Q.E.D.

In what follows, we let $S=U$; i.e., we assume that $S$ is a maximal $L_{0}$-submodule of $L_{-1}$ which is equal to its own annihilator.

LEMMA 8. $\operatorname{Ann}_{L_{1}} L_{-2}=\{0\}$.

Proof. Set $A=\operatorname{Ann}_{L_{1}} L_{-2}$. Then $\left[L_{-1},[S, A]\right] \subseteq\left[\left[L_{-1}, S\right], A\right]+\left[S,\left[L_{-1}, A\right]\right]$ $\subseteq\left[L_{-2}, A\right]+\left[S, L_{0}\right] \subseteq\{0\}+S=S$, and $\left[L_{-2},[S, A]\right]=\left[S,\left[L_{-2}, A\right]\right]=\{0\}$, so by Lemma $5,[S, A]=\{0\}$. Hence, $\left[S,\left[L_{-1}, A\right]\right] \subseteq\left[\left[S, L_{-1}\right], A\right]+\left[L_{-1},[S, A]\right] \subseteq$ $\left[L_{-2}, A\right]+\{0\}=\{0\}$, so $\{0\}=\left[L_{-1},\left[L_{-2}, A\right]\right]=\left[L_{-2},\left[L_{-1}, A\right]\right] \supseteq\left[\left[L_{-1}, S\right]\right.$, $\left.\left[L_{-1}, A\right]\right]=\left[\left[L_{-1},\left[L_{-1}, A\right]\right], S\right]$, so that $\left[L_{-1},\left[L_{-1}, A\right]\right] \subseteq \mathrm{Ann}_{L_{-1}} S=S$. Since we also have $\left[L_{-2},\left[L_{-1}, A\right]\right]=\left[L_{-1},\left[L_{-2}, A\right]\right]=\{0\}$, it follows from Lemma 5 that $\left[L_{-1}, A\right]=\{0\}$, so that by Lemma $1, A=\{0\}$. Q.E.D.

LEMMA 9. $\operatorname{Ann}_{L_{i}} L_{-2}=\{0\}, 1 \leqslant i \leqslant k$.

Proof. Since $\left[L_{-1}, \operatorname{Ann}_{L_{i}} L_{-2}\right] \subseteq \mathrm{Ann}_{L_{i-1}} L_{-2}, i>-1$, we prove this lemma by assuming the contrary and arriving at a contradiction using induction and Lemmas 1 and 8. Q.E.D.

We now assume that $L_{-1}=S+T$, where $S$ and $T$ are proper $L_{0}$-submodules of $L_{-1}$. 
LEMMA 10. $L_{-1}=S \oplus T$, where $S$ and $T$ are irreducible abelian $L_{0}$-submodules of $L_{-1}$.

Proof. By Lemma 4, $S$ and $T$ are abelian. If $v \in T \cap S$, then $\left[v, L_{-1}\right]=[v, T$ $+S] \subseteq[v, T]+[v, S]=\{0\}$, since both $T$ and $S$ are abelian. Hence, $L_{-1}=$ $T \oplus S$.

Let $S_{0}$ be a proper submodule of $S$. If we set $T_{0}=T \oplus S_{0}$, then we have by Lemma 4 that $T_{0}$ is abelian. Then for any $s_{0} \in S_{0}$, we have $\left[s_{0}, L_{-1}\right]=\left[s_{0}, T_{0}+S\right]$ $\subseteq\left[s_{0}, T_{0}\right]+\left[s_{0}, S\right]=\{0\}$, as before, so by Lemma $1, s_{0}=0$. Hence, $S$ can contain no proper submodule, so $S$ is irreducible. Similarly, $T$ is irreducible, also. Q.E.D.

LEMMA 11. $L_{-2}=[S, T]$.

Proof. By Lemma $1, L_{-2}=\left[L_{-1}, S\right]=[S \oplus T, S] \subseteq[S, S]+[T, S]=[T, S]$, since $S$ is abelian. Q.E.D.

LEMMA 12. $\operatorname{Ann}_{L_{0}} L_{-2} \cap \operatorname{Ann}_{L_{0}} S=\{0\}=\operatorname{Ann}_{L_{0}} S \cap \operatorname{Ann}_{L_{0}} T$.

Proof. Set $C=\mathrm{Ann}_{L_{0}} L_{-2} \cap \mathrm{Ann}_{L_{0}} S$. Then by definition, $\left[L_{-2}, C\right]=\{0\}$. Furthermore, $\left[L_{-1}, C\right]=[S \oplus T, C] \subseteq[S, C]+[T, C] \subseteq T$, which is abelian. Hence, by Lemma $5, C=\{0\}$. Set $D=\operatorname{Ann}_{L_{0}} S \cap \operatorname{Ann}_{L_{0}} T$. Then by Lemma 11, we have $\left[L_{-2}, D\right]=[[S, T], D]=[[S, D], T]+[S,[D, T]]=\{0\}$, so $D \subseteq C=$ \{0\}. Q.E.D.

LEMMA 13. $L_{2 i-1}=\left[L_{2 i}, S\right] \oplus\left[L_{2 i}, T\right], 0 \leqslant i \leqslant[k / 2]$.

Proof. Since by Lemma $2, L_{2 i-1}=\left[L_{2 i}, L_{-1}\right]$, we have by assumption that $L_{2 i-1} \subseteq\left[L_{2 i}, S\right]+\left[L_{2 i}, T\right]$. If $v \in\left[L_{2 i}, S\right] \cap\left[L_{2 i}, T\right]$, we have $v\left(\operatorname{ad} L_{-2}\right)^{i} \in S \cap$ $T=\{0\}$, so by Lemma 9 and induction, $v=0$. Q.E.D.

LeMma 14. Ann $\operatorname{Lin}_{2 i-1} S=\left[L_{2 i}, S\right], 0 \leqslant i \leqslant[k / 2]$.

Proof. Since $\left[\left[L_{2 i}, S\right], S\right]\left(\operatorname{ad} L_{-2}\right)^{i} \subseteq[S, S]=\{0\}$, we have by Lemma 9 and induction that $\left[\left[L_{2 i}, S\right], S\right]=\{0\}$. If $x \in\left(L_{2 i-1} \backslash\left[L_{2 i}, S\right]\right) \cap \operatorname{Ann}_{L_{2 i-1}} S$, then by Lemma 13, $x=v+y$, where $0 \neq v \in\left[L_{2 i}, T\right] \cap \mathrm{Ann}_{L_{2 i-1}} S$, and $y \in\left[L_{2 i}, S\right]$. Then $\{0\}=[v, S]\left(\operatorname{ad} L_{-2}\right)^{i}=\left[v\left(\operatorname{ad} L_{-2}\right)^{i}, S\right]$, so $v\left(\operatorname{ad} L_{-2}\right)^{i} \subseteq \operatorname{Ann}_{L_{-1}} S=S$ (see Lemma 10 and the remark following Lemma 7). But $v\left(\operatorname{ad} L_{-2}\right)^{i} \subseteq$ $\left[L_{2 i}, T\right]\left(\operatorname{ad} L_{-2}\right)^{i}=\left[L_{2 i}\left(\operatorname{ad} L_{-2}\right)^{i}, T\right] \subseteq T$, so $v\left(\operatorname{ad} L_{-2}\right)^{i} \subseteq S \cap T=\{0\}$. Thus, by Lemma 9 and induction, $v=0$. Q.E.D.

LEMMA 15. [[ $\left.\left.L_{2 i}, S\right],\left[L_{2 j}, S\right]\right]=\{0\}, 0<i, j<[k / 2]$.

Proof. [[ $\left.\left.L_{2 i}, S\right],\left[L_{2 j}, S\right]\right]\left(\operatorname{ad} L_{-2}\right)^{i+j} \subseteq[S, S]=\{0\}$, so by Lemma 9 and induction, [[ $\left.\left.L_{2 i}, S\right],\left[L_{2 j}, S\right]\right]=\{0\}$. Q.E.D.

LEMMA 16. $\left[L_{2 i},\left[L_{2 j}, S\right]\right] \subseteq \operatorname{Ann}_{L_{2(i+j)-1}} S$.

Proof. Since $\left[\left[L_{2 i},\left[L_{2 j}, S\right]\right], S\right]\left(\operatorname{ad} L_{-2}\right)^{i+j} \subseteq[S, S]=\{0\}$, we have by Lemmas 9 and 14 that $\left[L_{2 i},\left[L_{2 j}, S\right]\right] \subseteq \mathrm{Ann}_{L_{2(i+j)-1}} S$. Q.E.D.

LEMMA 17. If $k$ is odd, then $L_{k}$ annihilates $S$ or $T$. In the former case, we have $\left[L_{-2}, L_{k}\right] \subseteq\left[L_{k-1}, S\right]$. The latter case is similar. 
Proof. By Lemmas 3 and 2, we have that $\{0\} \neq\left[L_{0}, L_{k}\right]=\left[\left[L_{-1}, L_{1}\right], L_{k}\right]=$ $\left[L_{k-1}, L_{1}\right]$, so by Lemmas 2 and $13, L_{k}=\left[L_{k-1}, L_{1}\right]=\left[L_{k-1},\left[L_{2}, S\right] \oplus\left[L_{2}, T\right]\right] \subseteq$ $\left[L_{k-1},\left[L_{2}, S\right]\right]+\left[L_{k-1},\left[L_{2}, T\right]\right]$. By Lemma 16, $\left[L_{k-1},\left[L_{2}, S\right]\right] \subseteq \operatorname{Ann}_{L_{k}} S$, and since for ahy $i,-1 \leqslant i \leqslant k$, we have that $\left[L_{-2}, \operatorname{Ann}_{L_{i}} S\right] \subseteq \operatorname{Ann}_{L_{-2}} S$, we must have by Lemma 14 that $\left[L_{k-1},\left[L_{2}, S\right]\right]\left(\operatorname{ad} L_{-2}\right)^{((k+1) / 2)} \subseteq \operatorname{Ann}_{L_{-1}} S \subseteq S$. Similarly, $\left[L_{k-1},\left[L_{2}, T\right]\right]\left(\operatorname{ad} L_{-2}\right)^{((k+1) / 2)} \subseteq T$, so if $v \in\left[L_{k-1},\left[L_{2}, S\right]\right] \cap$ $\left[L_{k-1},\left[L_{2}, T\right]\right]$, then $v\left(\operatorname{ad} L_{-2}\right)^{((k+1) / 2)} \subseteq S \cap T=\{0\}$, so by Lemma 9 and induction, $v=0$. Thus, $L_{k}=\left[L_{k-1},\left[L_{2}, S\right]\right] \oplus\left[L_{k-1},\left[L_{2}, T\right]\right]$. But by Lemma $2, L_{k}$ is an irreducible $L_{0}$-module, so either $\left[L_{k-1},\left[L_{2}, S\right]\right]=\{0\}$ or $\left[L_{k-1},\left[L_{2}, T\right]\right]=\{0\}$. In the latter case, we have $\left[L_{-2}, L_{k}\right] \subseteq \operatorname{Ann}_{L_{k-2}} S=\left[L_{k-1}, S\right]$, by Lemma 14 . Q.E.D.

Proof of Theorem. We will assume that $\left[L_{k}, S\right]=\{0\}$, if $k$ is odd. The case where $\left[L_{k}, T\right]=\{0\}$ is symmetric. We set

$$
\begin{aligned}
& M_{-1}=L_{-2} \oplus S, \\
& M_{0}=T \oplus L_{0} \oplus\left[L_{2}, S\right], \\
& M_{i}=\left[L_{2 i}, T\right] \oplus L_{2 i} \oplus\left[L_{2 i+2}, S\right], \quad 1 \leqslant i<[k / 2],
\end{aligned}
$$

and

$$
M_{[k / 2]}=\left\{\begin{array}{l}
{\left[L_{k}, T\right] \oplus L_{k}, \quad \text { if } k \text { is even, }} \\
{\left[L_{k-1}, T\right] \oplus L_{k-1} \oplus L_{k}, \quad \text { if } k \text { is odd }}
\end{array}\right.
$$

Then by Lemmas 10 and $13, L=M=M_{-1} \oplus M_{0} \oplus \cdots \oplus M_{[k / 2]}$, and by Lemmas $6,14,15,16$, and $17,\left[M_{i}, M_{j}\right] \subseteq M_{i+j},-1<i, j<[k / 2]$. (If $k$ is odd, then both $\left[L_{2},\left[L_{k-1}, T\right]\right]$ and $\left[\left[L_{2}, T\right], L_{k-1}\right]$ annihilate $T$ by Lemma 16. As subspaces of $L_{k}$, they annihilate $S$. Hence, they are zero by Lemma 1.)

It remains to show that $M$ is irreducible and transitive. That $M$ is irreducible follows from Lemma 1 of [2]. We must now show that $M$ is transitive. By Lemma 9, we have that $\mathrm{Ann}_{M_{i}} M_{-1}=\{0\}$ for $i>0$. Moreover, we have that

$$
\begin{aligned}
\operatorname{Ann}_{M_{0}} M_{-1}= & \left(\operatorname{Ann}_{T} L_{-2} \cap \operatorname{Ann}_{T} S\right) \\
& \oplus\left(\operatorname{Ann}_{L_{0}} L_{-2} \cap \operatorname{Ann}_{L_{0}} S\right) \oplus\left(\operatorname{Ann}_{\left[L_{2}, S\right]} L_{-2} \cap \operatorname{Ann}_{\left[L_{2}, S\right]} S\right) .
\end{aligned}
$$

Because we have by Lemma 14 that $\mathrm{Ann}_{T} S \subseteq T \cap \mathrm{Ann}_{L_{-1}} S \subseteq T \cap S=\{0\}$, the first summand is zero. The second summand is zero by Lemma 12 . The third summand is contained in $\mathrm{Ann}_{L_{1}} L_{-2}$, which is zero by Lemma 8. To complete the proof, we have to check that $\mathrm{Ann}_{M_{-1}} M_{1}=\{0\}$ and $\mathrm{Ann}_{M_{0}} M_{1}=\{0\}$, which we do in the following proof, which is due to the referee, to whom the present author is most gratefu. That $\mathrm{Ann}_{M_{-1}} M_{1}=\{0\}$ follows from the irreducibility of $M_{-1}$ as an $M_{0}$-module and the fact that $\left[M_{-1}, M_{1}\right] \neq\{0\}$, which we have just demonstrated. Now let $M^{\prime}=\Sigma M_{i}^{\prime}$ be the subalgebra of $M$ generated by $M_{-1} \oplus M_{0} \oplus M_{1}$. Let $I$ be the ideal of $M^{\prime}$ generated by $\mathrm{Ann}_{M_{0}} M_{1}$. Then by the Poincaré-Birkhoff-Witt Theorem,

$$
\begin{aligned}
I & =U\left(M^{\prime}\right) \operatorname{Ann}_{M_{0}} M_{1}=U\left(M_{-1}\right) U\left(M_{0}\right) U\left(M_{1}^{\prime} \oplus \cdot M_{2}^{\prime} \oplus \cdots\right) \operatorname{Ann}_{M_{0}} M_{1} \\
& =U\left(M_{-1}\right) \operatorname{Ann}_{M_{0}} M_{1} \subseteq M_{-1} \oplus \operatorname{Ann}_{M_{0}} M_{1} .
\end{aligned}
$$


But $I \supseteq\left[I, M^{\prime}\right] \supseteq\left[M_{-1}, M_{1}\right] \supseteq\left[L_{-2},\left[L_{2}, T\right]\right] \supseteq T$ (by Lemmas 8 and 10 as $\left.\left[L_{-2},\left[L_{2}, T\right]\right]=\left[\left[L_{-2}, L_{2}\right], T\right]\right)$. Thus, $\left[T, L_{2}\right] \subseteq I \cap M_{1}=\{0\}$. Then by Lemmas 1 and 13 , we would have $L_{-1}=\left[L_{1}, L_{-2}\right]=\left[\left[L_{2}, S\right] \oplus\left[L_{2}, T\right], L_{-2}\right]=$ [[ $\left.\left.L_{2}, S\right], L_{-2}\right] \subseteq\left[\left[L_{2}, L_{-2}\right], S\right] \subseteq S$, which, by Lemma 10, contradicts our assumption that $L_{-1}$ is reducible. Q.E.D.

\section{REFERENCES}

1. T. B. Gregory, A characterization of the contact Lie algebras, Proc. Amer. Math. Soc. (to appear).

2. Simple Lie algebras with classical reductive null component, J. Algebra 63 (1980), 484-493.

3. V. G. Kac, The classification of the simple Lie algebras over a field with non-zero characteristic, Izv. Akad. Nauk SSSR Ser. Mat. 34 (1970), 385-408; English transl., Math. USSR Izv. 4 (1970), 391-413.

Department of Mathematics, The Oho State University at Mansfield, Mansfield, Ohio 44906 